\title{
The use of rhythmic auditory cues to influence gait in patients with Parkinson's disease, the differential effect for freezers and non-freezers, an explorative study
}

\author{
A. M. Willems MSc, K.U.Leuven, A. Nieuwboer, F. Chavret, K. Desloovere, R. \\ Dom, L. Rochester, D. Jones, G. Kwakkel \& E. Van Wegen
}

To cite this article: A. M. Willems MSc, K.U.Leuven, A. Nieuwboer, F. Chavret, K. Desloovere, R. Dom, L. Rochester, D. Jones, G. Kwakkel \& E. Van Wegen (2006) The use of rhythmic auditory cues to influence gait in patients with Parkinson's disease, the differential effect for freezers and non-freezers, an explorative study, Disability and Rehabilitation, 28:11, 721-728, DOI: $10.1080 / 09638280500386569$

To link to this article: http://dx.doi.org/10.1080/09638280500386569

\section{冓 Published online: 07 Jul 2009.}

Submit your article to this journal \lceil

Џ Article views: 618

Q View related articles $₫$

4 Citing articles: 1 View citing articles 준 


\title{
The use of rhythmic auditory cues to influence gait in patients with Parkinson's disease, the differential effect for freezers and non-freezers, an explorative study
}

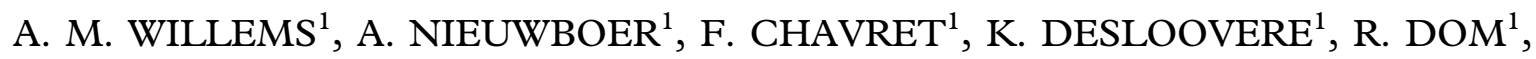 \\ L. ROCHESTER ${ }^{2}$, D. JONES ${ }^{2}$, G. KWAKKEL ${ }^{3}$, \& E. VAN WEGEN ${ }^{3}$ \\ ${ }^{1}$ Katholieke Universiteit Leuven, Belgium, ${ }^{2}$ Northumbria University Newcastle, UK, and ${ }^{3}$ Vrije Universiteit Medisch Centrum \\ Amsterdam, The Netherlands
}

Accepted September 2005

\begin{abstract}
Purpose. To study the effect of rhythmic auditory cues on gait in Parkinson's disease subjects with and without freezing and in controls.

Method. A volunteer sample of 20 patients (10 freezers, 10 non-freezers) and 10 age-matched controls performed five randomized cued walking conditions in a gait-laboratory. Auditory cues were administered at baseline frequency, at an increased step frequency of 10 and $20 \%$ above baseline and at a decreased step frequency of 10 and $20 \%$ below baseline. Mean step frequency, walking speed, stride length and double support duration were collected.

Results. Rhythmical auditory cueing induced speed changes in all subjects. Stride length was not influenced by rhythmical auditory cues in controls, whereas patients showed a larger stride length in the $-10 \%$ condition $(p<0.01)$. Freezers and non-freezers showed the same response to rhythmical auditory cues. Within group analysis for stride length showed different cueing effects. Stride length decreased at the $+10 \%$ condition for freezers $(p<0.05)$, whereas it increased for nonfreezers.

Conclusions. This study points to fact that physiotherapists might need to carefully adjust the cueing frequency to the needs of patients with and without freezing. On the basis of the present results we recommend to lower the frequency setting for freezers, whereas for non-freezers an increase of up to $+10 \%$ may have potential therapeutic use.
\end{abstract}

Keywords: Parkinson's disease, cueing, gait, freezing

\section{Introduction}

Most people with Parkinson's disease (PD) experience walking difficulties as the disease progresses [1]. A slow, short-stepped gait with a rigid trunk and postural instability accompany PD and cause important functional loss [2,3]. Furthermore festination and freezing hinder normal ongoing gait and often cause falls $[2,4,5]$. Studies have demonstrated that several motor tasks including gait can be improved when cues are provided [6-8]. A variety of specific stimuli of a visual [9] or auditory [10-13] nature can act as cues and improve gait. According to Morris et al. cues should preferably be aimed at enlarging the patient's stride length to have a maximum impact on normalizing Parkinsonian gait $[2,8]$. As Rhythmic Auditory Cues (RAC) direct the temporal regulation of a movement, the effect on stride length might be less evident.

McIntosh et al. concluded that patients were able to follow an increased step frequency both during the on and off phase of the medication cycle by calculating synchronization errors for an increased cueing rhythm [12]. Several recent experiments showed that providing RAC at an increased step frequency of $10-15 \%$ of patients' natural step frequency had an immediate effect on step frequency, walking speed and stride length $[10,11,13]$. Despite these positive results, the effects of RAC on stride length remain inconsistent. Morris et al. did 
not find an increase of stride length when patients' step frequency was increased towards the step frequency of control subjects [2]. A recent study by Howe et al. studying the effect of increasing and decreasing subject's step frequency using RAC in early PD, did not show any effect on stride length [14]. As contradictory findings are reported, further research seems warranted. In addition the effects of imposing a decreased step frequency should be explored more extensively, as the relative step frequency (measured at the same speed) tends to be increased in PD when compared with controls [2], supporting the argument for normalization of gait by lowering the cueing frequency. There are, however, indications that patients find it difficult to follow decreased cueing rhythms [15]. In a recently constructed physical therapy management-model, different therapy approaches are advised for influencing gait hypokinesia and gait akinesia (freezing) in PD [16]. As the external temporal character of RAC might act as a counterbalancing system for the disturbed internal timekeeper-function of the basal ganglia [17], it may be especially suited to discourage freezing. It has recently been shown that patients with PD exhibiting freezing symptoms have an increased variability of step time during normal gait, reflecting a disturbed temporal organization of walking [18]. A recent study by Nieuwboer et al. has revealed a disrupted temporal organization at the onset of freezing, appearing as a hastening of stepping [19]. An anecdotal finding reported by McIntosh et al. [12] suggests the use of RAC to overcome and avoid freezing episodes. Thus far, cueing studies have not differentiated between subjects presenting with hypokinetic and akinetic (freezing) gait.

This present study attempts to clarify some of the above-mentioned unresolved issues with regard to cueing that is: Whether RAC can influence stride length, whether the effects are different for freezers and non-freezers, whether the enforced step frequency should be above or below the patient's self chosen step frequency and whether patients are able to synchronize their stepping frequency to the RAC.

\section{Methods}

Subjects

Twenty patients with PD and 10 age-matched controls were recruited. Patients, who indicated to the referring neurologist (University hospital Leuven, Gasthuisberg) that they were willing to take part in a gait experiment, were invited if they met the following inclusion criteria: (i) diagnosis of idiopathic Parkinson's disease, (ii) disease severity on the modified Hoehn \& Yahr scale [20] ranging between stage I. 5 and IV during the 'on' phase, (iii) ability to walk for $10 \mathrm{~m}$ repeatedly, and (iv) stable medication phase during the time of testing (patients should not rate themselves in the 'off' phase during the testing period) [21]. They were excluded if they: (i) presented with co-morbidity limiting walking ability, (ii) had had brain surgery in the past, (iii) had serious hearing deficits, (iv) presented with severe dyskinesias (score $>1$ Modified Dyskinesia Scale) [22] during the testing period, or (v) had a score of 24 or lower on the Mini Mental State Examination (MMSE) [23]. Patients were on a stable medication regimen. All but one was taking Levodopa. Most subjects $(90 \%)$ were also medicated with dopamine agonists. Some took COMT inhibitors $(45 \%)$ and a minority took anti-cholinergic medication $(15 \%)$ or amantadine $(15 \%)$.

Two distinct patient groups (10 freezers and 10 non-freezers) were recruited. Subjects' age and results on the MMSE were recorded, as well as other descriptors such as disease duration, the modified Hoehn \& Yahr scale, the motor part of the Unified Parkinson's Disease Rating Scale (UPDRS) [24] and the Freezing of Gait questionnaire (FoGQ) [25]. Freezers were defined as patients with freezing episodes occurring at least once a week, (minimum score of 2 on item 3 of the FoGQ). The age-matched controls were volunteers, in most cases the patient's carers. Controls showing (i) co-morbidity limiting walking ability, (ii) a serious hearing deficit, or (iii) a MMSE score of 24 or lower were excluded. A control group was included to be able to interpret the pattern of responses of freezers and non-freezers to various cueing frequencies. All subjects gave their written and informed consent according to the Helsinki Declaration and ethical approval was received from the ethical committee of the Catholic University Leuven.

\section{Apparatus}

Gait analysis was performed with an eight camera VICON data capturing system (Vicon Motion Systems, workstation 612), positioned around an $8 \mathrm{~m}$ walkway. Reflective markers $(14 \mathrm{~mm}$ in diameter) were placed bilaterally on the anterior superior iliac spine, the sacrum, the mid-thigh, the lateral femur condyle, the mid-shank, the lateral malleolus, the dorsal aspect of the foot between the second and third metatarsal head, and on the calcaneus. A metronome, providing the RAC, was integrated into the measuring system. A metronome, with an infrared light, flashing simultaneously with the auditory beat, was used. This enabled registration of the metronome beat (flash) with the infrared cameras of the VICON system. 


\section{Test-protocol}

All patients were tested during the 'on' phase of the medication cycle, approximately $1-2 \mathrm{~h}$ after their midday medication intake. To check whether patients remained in a stable medication phase, they were regularly asked to indicate whether they were 'on' or 'off' on a visual analogue scale developed by Nieuwboer et al. [21]. None of the patients indicated an 'off' phase during the test period. Subjects were asked to walk $8 \mathrm{~m}$ along the walkway under five randomized cueing conditions. The cueing conditions were preceded by one normal walking condition at preferred speed. From this, the preferred step frequency was determined, referred to as baseline. Auditory cues were administered at baseline frequency, at an increased step frequency of $10 \%$ and $20 \%$ above baseline and at a decreased step frequency of $10 \%$ and $20 \%$ below baseline. Cueing conditions were offered in a randomized order to prevent cross-over effects. Subjects were instructed to synchronize their foot contact with the metronome beat. Each of the five cueing conditions was performed three times, preceded by one practice trial. None of the freezers froze during the gait laboratory tests.

\section{Data processing}

Metronome beats and gait cycle events (toe off and initial foot contact) were defined manually, using Vicon Clinical Manager software (Oxford Metrics, Oxford, UK). This procedure is known to have a good inter-rater reliability in Parkinsonian gait (Intra-Class-Correlation between 0.93 and 1) [19]. The measurement area was located in the middle of the $8 \mathrm{~m}$ walkway to rule out possible acceleration and deceleration effects, enabling the recording from 4-6 steps, depending on the person's step length. For each cueing condition the spatiotemporal variables (step frequency, walking speed, stride length and double support time) of the three walking trials, using as many steps as possible, were averaged for the left and right side separately. Double support time was expressed as a percentage of the gait cycle duration. Differences between the disease dominant and non-dominant side were explored before starting final data analysis and showed no differences. Therefore, results are presented for the disease dominant side only in PD subjects. Consequently, a one-side analysis for controls (left side) was used. To measure the ability to walk synchronized to a metronome beat a synchronization error was calculated. Synchronization error was expressed as the absolute time difference between the occurrence of metronome beat $(\mathrm{M})$ and foot contact $(\mathrm{F})$, normalized to step duration. ( $|\mathrm{F}-\mathrm{M}|{ }^{\star} 100 /$ step duration). Synchronization error was calculated for each walking trial and averaged for each cueing condition.

\section{Data analysis}

$T$-tests and Mann-Whitney $\mathrm{U}$ tests were used to analyse descriptor variables. For exploration of spatiotemporal data, a multivariate model of repeated measures was used to study the five different cueing conditions, within and between the two groups using an unstructured or more simplified covariance matrix in SAS (statistical analysis software). Two successive 2-groups comparisons were conducted. Only successive cueing conditions were compared for the within group analysis. As we were interested in the error levels of decreased and increased cueing frequency the $-10 \%$ and the $-20 \%$ and the $+10 \%$ and $+20 \%$ conditions were pooled and analysed together. Each hypothesis was tested at the 0.05 level of significance. As this study was exploratory in nature, Bonferroni corrections were not carried out.

\section{Results}

The PD and control group were comparable for age (PD: 64.5 years \pm 7.5 , Control: 63.6 years \pm 5.0, $p>0.05$ ) and cognitive function (MMSE: PD: $27.7 \pm 2.3$, Control: $28.9 \pm 0.7, p>0.05)$. The patient subgroups (see Table I) were comparable for MMSE $(p>0.05)$ and disease severity, as rated with the motor part of the UPDRS $(p>0.05)$ and

Table I. Characteristics of non-freezers and freezers.

\begin{tabular}{|c|c|c|c|c|c|c|}
\hline & Age (y) & Disease Duration (y) & FoGQ & MMSE & UPDRS III (on) & $\mathrm{H} \& \mathrm{Y}$ (on) \\
\hline Non-freezers & $60.6(48-67)^{\star}$ & $6.2(2.5-11)^{\star}$ & $5.5^{\star \star}$ & 28.5 & 24.7 & 2.7 \\
\hline$S D$ & 6.2 & 3 & 4.9 & 2.2 & 12.6 & 0.6 \\
\hline Freezers & $68.4(58.5-80)$ & $11.8(0-18)$ & 16.1 & 26.9 & 27.2 & 2.8 \\
\hline$S D$ & 6.9 & 5.7 & 4.3 & 2.1 & 11.3 & 0.6 \\
\hline
\end{tabular}

The mean and standard deviation (SD) are presented. Numbers between brackets represent the range of data distribution. ${ }^{\star} \mathrm{Groups}$ statistically different at $p<0.05$ ( $t$-test). ${ }^{\star \star}$ Groups statistically different at $p<0.01$ ( $t$-test). 
the modified Hoehn and Yahr-scale $(p>0.05)$. However the freezer group was older $(p<0.05)$, experienced longer disease duration $(p<0.05)$ and had a higher FoGQ-score $(p<0.01)$ than the nonfreezer group.

\section{Spatiotemporal data}

Non-cued walking. When PD subjects walked at preferred walking speed, they proved to walk slower $(p<0.01)$ and with shorter strides $(p<0.01)$ than controls (see Table II). In addition, step frequency was significantly reduced $(p<0.05)$, whereas double support time was significantly increased $(p<0.05)$ compared to the control group.

Freezers and non-freezers did not differ significantly for any of the spatiotemporal data.

Cued walking. In all cueing conditions PD subjects walked significantly slower $(p<0.01)$ and used shorter strides than controls $(p<0.01)$. Further- more, the step frequency remained lower than controls in the cueing conditions $(p<0.05)$ but the difference was not significant in the $+10 \%$ condition. The double support time was significantly longer for PD than for controls in all conditions $(p<0.05)$ (see Table III).

When comparing the non-cued walking with the cueing condition at baseline, a significant increase of step frequency was observed for the PD group $(p<0.05)$, as well as for the control group $(p<0.01)$. None of the other parameters changed significantly with cueing at baseline in comparison to the normal walking condition without a cue.

As can be observed in Table III, step frequency increased with every $10 \%$ increment of the cueing rhythm for both groups $(p<0.01)$. The same pattern was observed in walking speed for both groups $(p<0.05)$, except between the $+10 \%$ and $+20 \%$ conditions for PD patients when speed remained unchanged. Stride length did not change across conditions for control subjects. PD subjects showed

Table II. Spatiotemporal data for controls, patients, and patients subgroups: Freezers and non-freezers in the non-cued walking condition.

\begin{tabular}{lcccc}
\hline & Step frequency (step/min) & Speed (m/sec) & Stride length (m) & Double support (\%GC) \\
\hline Controls & 114.90 & 1.20 & 1.26 & 23.50 \\
$S E$ & 1.91 & 0.03 & 0.03 & 0.72 \\
PD & $106.45^{\star}$ & $0.88^{\star}$ & $0.97^{\star}$ & $26.43^{\star}$ \\
$S E$ & 2.96 & 0.06 & 0.05 & 0.94 \\
Non-Freezers & 106.24 & 0.91 & 1.01 & 26.46 \\
SE & 3.74 & 0.07 & 0.06 & 1.17 \\
Freezers & 106.61 & 0.85 & 0.93 & 26.77 \\
SE & 4.62 & 0.08 & 0.07 & 1.46 \\
\hline
\end{tabular}

The estimated mean (linear mixed model) and the Standard Error (SE) are presented. Double support is presented as a percentage of the gait cycle (\% GC). ${ }^{\star} \mathrm{PD}$ and control statistically different $(p<0.05)$.

Table III. Spatiotemporal data of controls and PD in the five cueing conditions.

\begin{tabular}{|c|c|c|c|c|c|}
\hline & \multicolumn{5}{|c|}{ Cueing conditions } \\
\hline & $-20 \%$ & $-10 \%$ & Baseline & $10 \%$ & $20 \%$ \\
\hline \multicolumn{6}{|c|}{ Step frequency (step/min) } \\
\hline Controls & $97.2(2.1)$ & $108.7(1.9)^{\star \star}$ & $118.1(1.96)^{\star \star}$ & $126.8(2.8)^{\star \star}$ & $136.4(3.1)^{\star \star}$ \\
\hline PD & $88.6(3.0)$ & $98.7(3.1)^{\star \star}$ & $108.6(3.3)^{\star \star}$ & $118.4(3.2)^{\star \star}$ & $125.7(3.6)^{\star \star}$ \\
\hline \multicolumn{6}{|c|}{ Speed $(\mathrm{m} / \mathrm{sec})$} \\
\hline Controls & $1.00(0.04)$ & $1.14(0.03)^{\star \star}$ & $1.22(0.04)^{\star \star}$ & $1.33(0.04)^{\star \star}$ & $1.40(0.05)^{\star}$ \\
\hline PD & $0.73(0.05)$ & $0.85(0.05)^{\star \star}$ & $0.90(0.06)^{\star \star}$ & $0.98(0.06)^{\star}$ & $0.99(0.07)$ \\
\hline \multicolumn{6}{|c|}{ Stride length (m) } \\
\hline Controls & $1.24(0.04)$ & $1.26(0.03)$ & $1.24(0.04)$ & $1.26(0.04)$ & $1.23(0.05)$ \\
\hline PD & $0.97(0.04)$ & $1.02(0.04)^{\star \star}$ & $0.98(0.04)^{\star \star}$ & $0.98(0.05)$ & $0.93(0.04)$ \\
\hline \multicolumn{6}{|c|}{ Double support (\%GC) } \\
\hline Controls & $23.69(0.87)$ & $23.27(0.62)$ & $22.39(0.59)$ & $21.86(0.62)$ & $21.68(0.70)$ \\
\hline PD & $26.84(0.84)$ & $25.52(0.82)^{\star}$ & $24.86(0.80)$ & $25.11(0.80)$ & $24.88(0.87)$ \\
\hline
\end{tabular}

The estimated mean (linear mixed model) and the Standard Error (SE) are presented. Double support time is presented as a percentage of the gait cycle (\% GC). Significant differences for within group comparisons are presented, with regards to the preceding cueing condition. ${ }^{\star} p<0.05,{ }^{\star \star} p<0.01$. In between group comparisons are not presented. 
a significantly larger stride length in the $-10 \%$ condition compared with the baseline condition $(p<0.01)$ and compared with the $-20 \%$ condition $(p<0.01)$.

In the control group, double support time values did not differ between the different cueing conditions. In the PD group double support time significantly decreased in the $-10 \%(p<0.05)$ in comparison with the $-20 \%$ condition.

Only for non-freezers, cueing at baseline resulted in a significant increase of step frequency when compared with non-cued walking $(p<0.01)$. Freezers and non-freezers did not differ on any of the spatiotemporal data across cueing conditions. (see Table IV).

Table IV shows that step frequency increased with each increasing cueing rhythm in both groups $(p<0.05)$. Across conditions, an increase in cueing rhythm was combined with an increase of walking speed $(p<0.05)$ except in two cases. An increase of step frequency between the baseline condition and $+10 \%$ was not associated with an increased speed for the freezers. Secondly, the non-freezers' speed reached a plateau between the $+10 \%$ and $+20 \%$ condition. The interaction for speed between cueing conditions and group was significant $(p<0.05)$. For non-freezers, stride length was larger in the $-10 \%$ condition in comparison with the $-20 \%(p<0.05)$ and baseline condition $(p<0.01)$ (see Figure 1).

The same pattern was observed for freezers but the differences failed to reach significance. The different statistical outcome can be explained by the fact that the applied statistics (mixed model) relied on estimations of the difference scores rather than on the group means and standard deviations.
A significantly decreased stride length for freezers was observed in the $+10 \%$ condition when compared with baseline $(p<0.05)$. Although not significant, the opposite pattern was observed in the non-freezer group. The interaction between cueing conditions and group proved to be significant for stride length $(p<0.05)$. For freezers double support times did not differ across cueing conditions. Non-freezers on the other hand showed a significant increased double support time in the $-20 \%$ condition in comparison with the $-10 \%$ condition $(p<0.05)$.

\section{Synchronization error}

Synchronization error is presented in Table V. Parkinson's disease subjects made larger errors than controls in the lower than baseline frequencies $(p<0.05)$. The reverse pattern was observed in the higher frequencies $(p<0.05)$. At baseline the two groups did not differ significantly. The interaction between cueing conditions and group was significant $(p<0.01)$.

The within-group analysis revealed a larger error at the lower cueing conditions compared with baseline for PD $(p<0.01)$. However, there was no significant difference between the errors produced at baseline and at the higher than baseline frequencies. For controls no significant differences were observed between conditions.

Freezers made smaller errors than non-freezers. This difference was only significant for the lower than baseline conditions $(p<0.05)$ (see Table V). The within-group analysis revealed that larger errors occurred in the lower conditions than at baseline for both groups $(p<0.05)$. However, in comparison

Table IV. Spatiotemporal data of non-freezers and freezers in the 5 cueing conditions.

\begin{tabular}{|c|c|c|c|c|c|}
\hline & \multicolumn{5}{|c|}{ Cueing conditions } \\
\hline & $-20 \%$ & $-10 \%$ & Baseline & $10 \%$ & $20 \%$ \\
\hline \multicolumn{6}{|c|}{ Step frequency (step/min) } \\
\hline Non-Freezers & $89.3(3.7)$ & $99.2(4.0)^{\star \star}$ & $109.1(3.8)^{\star \star}$ & $119.8(3.5)^{\star \star}$ & $125.6(4.1)^{\star}$ \\
\hline Freezers & $87.9(4.7)$ & $97.9(4.7)^{\star \star}$ & $108.1(5.3)^{\star \star}$ & $117.1(5.3)^{\star \star}$ & $125.8(5.9)^{\star \star}$ \\
\hline \multicolumn{6}{|c|}{ Speed $(\mathrm{m} / \mathrm{sec})$} \\
\hline Non-Freezers & $0.74(0.08)$ & $0.86(0.08)^{\star \star}$ & $0.91(0.09)^{\star \star}$ & $1.05(0.08)^{\star \star}$ & $1.03(0.10)$ \\
\hline Freezers & $0.72(0.06)$ & $0.84(0.07)^{\star \star}$ & $0.90(0.08)^{\star}$ & $0.90(0.09)$ & $0.95(0.08)^{\star}$ \\
\hline \multicolumn{6}{|c|}{ Stride length (m) } \\
\hline Non-Freezers & $0.97(0.07)$ & $1.02(0.06)^{\star}$ & $0.98(0.07)^{\star \star}$ & $1.04(0.06)$ & $0.97(0.07)$ \\
\hline Freezers & $0.98(0.05)$ & $1.02(0.04)$ & $0.98(0.05)$ & $0.91(0.06)^{\star}$ & $0.90(0.05)$ \\
\hline \multicolumn{6}{|c|}{ Double support (\%GC) } \\
\hline Non-Freezers & $27.44(1.29)$ & $25.94(1.22)^{\star}$ & $25.03(1.30)$ & $24.95(1.29)$ & $24.88(1.35)$ \\
\hline Freezers & $26.25(1.04)$ & $25.10(1.08)$ & $24.70(0.94)$ & $25.28(0.95)$ & $24.89(1.08)$ \\
\hline
\end{tabular}

The estimated mean (linear mixed model) and the Standard Error (SE) are presented. Double support time is presented as a percentage of the gait cycle (\% GC). Significant differences for within group comparisons are presented, with regards to the preceding cueing condition. ${ }^{\star} p<0.05,{ }^{\star \star} p<0.01$. In between group comparisons are not presented. 


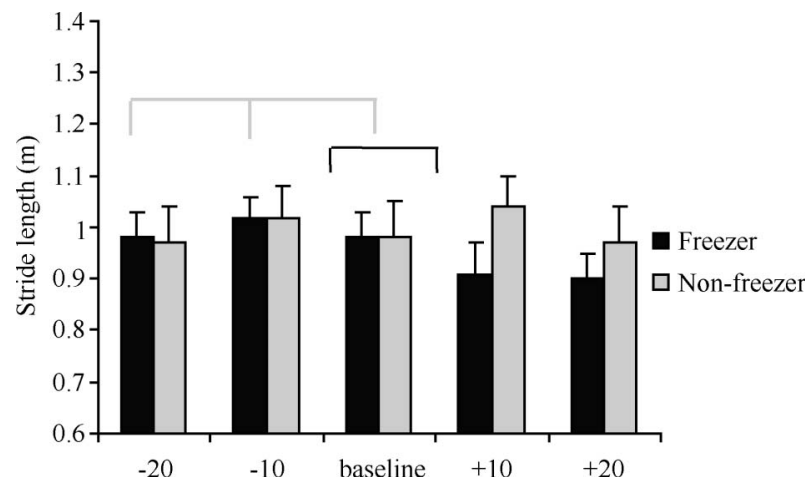

Figure 1. Influence of cueing frequency on stride length for nonfreezers and freezers. The estimated mean (linear mixed model) and the Standard Error (SE) are presented. Horizontal braces represent statistically significant difference between the conditions $p<0.05$.

Table V. Synchronization error for cueing at baseline and pooled high and low cueing frequencies.

\begin{tabular}{|c|c|c|c|}
\hline \multirow{2}{*}{$\begin{array}{l}\text { Synchronisation } \\
\text { Error }\end{array}$} & \multirow[b]{2}{*}{ Low } & \multicolumn{2}{|c|}{ Cueing conditions } \\
\hline & & Baseline & High \\
\hline Controls & $15.35(1.83)$ & $14.96(1.81)$ & $17.65(1.33)$ \\
\hline PD & $22.42(1.54)$ & $15.89(2.38)^{\star}$ & $12.97(1.72)$ \\
\hline Non-freezers & $25.44(2.16)$ & $18.25(3.94)^{\star}$ & $13.62(2.91)$ \\
\hline Freezers & $19.40(1.73)$ & $13.54(2.46)^{\star}$ & $12.61(1.79)$ \\
\hline
\end{tabular}

The estimated mean (linear mixed model) and the Standard Error (SE) are presented. Significant differences for within group comparisons are presented, with regards to the preceding cueing condition. ${ }^{\star} p<0.05$. In between group comparisons are not presented.

with the higher conditions, the bigger errors in baseline were not significant for both groups.

\section{Discussion}

This study aimed to investigate some unresolved questions about the effect of RAC in PD and the differential effect in freezers and non-freezers. This experiment demonstrates an effect of RAC on stride length for PD only. Stride length did not change across conditions for controls. In contrast to previous studies $[10,11,13]$, stride length for PD did not increase in the $+10 \%$ condition, but it did in the $-10 \%$ condition. The $-10 \%$ condition enforces a slower walking, which might unlock the normal compensatory interplay between gait parameters. Morris et al. showed that PD patients increase their step frequency to speed up their gait, while stride length remains small [8]. While the $-10 \%$ condition allows for a slowing down of gait speed it may enable patients to increase their stride length. This mechanism may be similar to the speed-accuracy trade-off
[26], whereby as speed decreases accuracy will improve as patients have more time for accurate performance. The suggested trade-off between walking speed and stride length raises the question of why similar results were not found for the $-20 \%$ condition.

The $-10 \%$ condition may be considered as still falling within a normal range of speeds for a PD population, whereas the $-20 \%$ condition could represent an abnormally slow walking speed, in which the speed-stride length trade-off may no longer apply. Moreover, as the $-20 \%$ condition represents a very slow walking rhythm it might involve an additional challenge to already compromised postural stability, in which too large a step length could be detrimental for maintaining balance.

This experiment shows a marked stride length decrease for PD in the $+20 \%$ condition. This finding might explain the ceiling effect of velocity for patients at this frequency. Perhaps they reached a limit beyond which they could no longer increase speed. In contrast, controls were able to increase their walking speed with each increasing step frequency. Morris et al. [27] modulated the step frequency for PD and controls ranging from 40 up to 180 steps/min (with a stepwise increase of $10 \mathrm{steps} / \mathrm{min}$ ) with the intention to depict the stride length-cadence relationship. Stride length increased linearly with cadence for both PD and controls up to a critical point (120 steps/min for PD, 130 steps/min for controls) where increase of cadence was followed by a decreased stride length. In this experiment, this critical point may have been reached near the $+20 \%$ condition (126 steps/min for PD). Our results do not confirm the linear trend as described by Morris et al., which could be related to the smaller number of measurements in this study compared with Morris's study [27]. When providing auditory cues at baseline both patients and controls increased their step frequency compared with the reference condition (walking at preferred speed). At first sight, this result could appear surprising as cueing in the baseline condition does not give any additional information on how to change the gait parameters. A possible explanation for this result could be that RAC have a general stimulating effect as was hypothesized by McCoy et al. [11]. However, this potentially stimulating effect on step frequency did not have any impact on speed. In addition, this effect was not apparent in freezers, a finding which is difficult to explain.

This experiment included both freezers and nonfreezers to detect a possible differential effect of RAC. The two subgroups proved to be comparable for disease severity and initial gait parameters. This can be explained by the fact that the tests were done in the 'on' period of the medication cycle [28]. 
Despite the comparability of the two subgroups at onset and during the cueing conditions, freezers and non-freezers reacted differently to cueing at an increased step frequency when within-group comparisons were analysed. At an increased step frequency of $+10 \%$, stride length increased for the non-freezers whereas it decreased for the freezers. The stride length increase for non-freezers was associated with an increased walking speed found at $+10 \%$. Freezers on the other hand, failed to increase walking speed in the same condition. To explain the different responses between freezers and non-freezers, it is attractive to refer back to the earlier discussed mechanisms of trade-off on the one hand and the linear stride-cadence relationship [27] on the other. The trade-off mechanism, whereby high frequencies and faster gait can be obtained at the cost of stride length, may be more at play in freezers. This group was able to increase stride length in the lower frequencies, except for the $-20 \%$ condition. Non-freezers on the other hand may fit better into the linear model, as they were able to increase stride length in the higher frequencies. However, the stride increase at $-10 \%$ is not in line with this explanation. Further research is needed to clarify this issue. One may suspect that previous studies on RAC included mainly non-freezers, which explains the stride length increase found at an increased step frequency of $+10 \%$ or $+15 \%[10,11,13]$.

Double support time showed a decline with each increasing cueing frequency for controls only. This decline may be expected in response to increasing speed, as this eventually evolves to running without double support but with double float phases. Freezers did not follow this trend in the higher cueing conditions and showed a non-significant increased double support time at $+10 \%$. This increase might be a compensation for balance problems in the higher cueing conditions. This balance protecting mechanism might slow down the walking and free less time to make bigger steps.

This experiment revealed no systematical changes in synchronization error for the different cueing frequencies in controls. Patients however, made larger synchronization errors at lower cueing frequencies. As patients are probably more used to a relatively increased stepping rate, a lower frequency might be more challenging, explaining the higher error levels. These findings are consistent with those of Ebersbach et al. who found increased stride variability at a decreased stepping rate of $20 \%$ [15]. The discrepancy between patients and controls might be associated with a different degree of attentiveness to the enforced step frequency. It is possible that patients rely more on the cue as a possible guidance for gait. McIntosh and colleagues also focused on the ability of patients to follow an auditory rhythm, albeit for only a $10 \%$ increment of the baseline step frequency [12]. Contrary to our results, they found an increase of synchronization error with an increase of the cueing frequency. This contradiction may be explained by the fact that in the current study, baseline was calculated from walking at a comfortable speed, whereas McIntosh et al. determined the baseline to be the maximum walking speed [12]. As a consequence, in the study of McIntosh et al. some subjects may have reached a limit beyond which they were not able to follow the rhythm.

Both freezers and non-freezers followed the general trend of making larger mistakes in synchronizing to lower frequencies than to higher ones. Surprisingly, as freezers have more problems with the timing of walking, the freezer group made smaller errors than the non-freezers especially in synchronizing to the lower cueing rhythms. Perhaps freezers are more dependent on cueing and therefore make smaller mistakes than non-freezers, because they experience timing difficulties during normal walking.

In conclusion, this study presents novel findings in the domain of auditory cueing as a way to influence gait in PD. First of all, the importance of frequency setting is emphasised. On the basis of the current results we can recommend guidelines for RAC depending on the therapy goals. An increased cueing frequency of $10 \%$ results in normalised step frequency values for the whole PD group. Although normal values for speed and stride length could not be obtained with RAC, the $+10 \%$ condition seems best to increase speed, at least in non-freezers, whereas the $-10 \%$ condition is better suited to achieve stride length enlargement alone. Secondly, this study has shown that it is important to target cueing frequency differently in freezers and nonfreezers. On the basis of the present results we recommend to lower the frequency setting for freezers, whereas for non-freezers an increase of up to $+10 \%$ still has potential therapeutic use. As this study is conducted on a small sample of patients, the results need to be interpreted with caution. Future studies also need to address whether patients are able to adapt their gait in the off-state in response to cueing. This may also reveal whether actual freezing episodes can be prevented or shortened by RAC. Furthermore, it is paramount to investigate the effects of RAC on the kinematic, kinetic and EMG profiles of gait. This might lead to a better understanding of the underlying mechanism of RACinduced gait changes and explain why increased stride length is obtained when slowing down the step frequency. The present study showed that RAC induced relatively small alterations of gait parameters. To achieve a clinically relevant effect on gait, long-term use and training with cues is 
indicated. Currently, a study into the long-term effects of auditory cueing is being carried out using the guidelines based on the present work.

\section{Acknowledgements}

Financial support is gratefully acknowledged from the European Commission. Framework V funding; QLRT-2001-00120. RESCUE-project.

\section{References}

1. Nieuwboer A, De Weerdt W, Dom R, Lesaffre E. A frequency and correlation analysis of motor deficits in Parkinson patients. Disabil Rehab 1998;20(4):142-150.

2. Morris ME, Ianesk R, Matyas TA, Summers JJ. The pathogenesis of gait hypokinesia in Parkinson's disease. Brain 1994:117(Pt 5):1169-1181.

3. Koller WC, Glatt S, Vetere-Overfield B, Hassanein R. Falls and Parkinson's disease. Clin Neuropharmacol 1989:12(2):98-105.

4. Giladi N. Gait disturbances in advances stages in Parkinson's disease. Adv Neurol 2001:86:273-278.

5. Gray P, Hildebrand K. Fall risk factors in Parkinson's disease. J Neurosci Nurs 2000:32(4):222-228.

6. Marchese R, Diverio M, Zucchi F, Lentino C, Abbruzzese G. The role of sensory cues in the rehabilitation of Parkinsonian patients: A comparison of two physical therapy protocols. Mov Disord 2000:15(5):879-883.

7. Rubinstein TC, Giladi N, Hausdorff JM. The power of cueing to circumvent dopamine deficits: A review of physical therapy treatments of gait disturbances in Parkinson's disease. Mov Disord 2002:17(6):1148-1160.

8. Morris ME, Ianesk R, Matyas TA, Summers JJ. Stride length regulation in Parkinson's disease Normalization strategies and underlying mechanisms. Brain 1996:119(Pt 2):551-568.

9. Lewis GN, Byblow WD, Walt SE. Stride length regulation in Parkinson's disease: The use of extrinsic, visual cues. Brain 2000:123(Pt 10):2077-2090.

10. Freedland RL, Festa C, Sealy M, et al. The effects of pulsed auditory stimulation on various gait measurements in persons with Parkinson's Disease. Neurorehabilitation 2002: 17(1):81-87.

11. McCoy RW, Kohl RM, Elliott SM, Joyce AS. The impact of auditory cues on gait control of individuals with Parkinson's disease. J Human Move Stud 2002:42:229-236.

12. McIntosh GC, Brown SH, Rice RR, Thaut MH. Rhythmic auditory-motor facilitation of gait patterns in patients with Parkinson's disease. J Neurol Neurosurg Psychiatr 1997: 62(1):22-26.

13. Thaut MH, McIntosh GC, Rice RR, Miller RA, Rathbun J, Brault JM. Rhythmic auditory stimulation in gait training for Parkinson's disease patients. Mov Disord 1996:11(2):193200.
14. Howe E, Lövgreen B, Cody FWJ, Ashton VJ, Oldham JA Auditory cues can modify the gait of persons with early-stage Parkinson's disease: A method for enhancing parkinsonian walking performance? Clin Rehabil 2003:17(4):363-367.

15. Ebersbach G, Heijmenberg M, Kindermann L, Trottenberg $\mathrm{T}$, Wissel J, Poewe W. Interference of rhytmic constraints on gait in healthy subjects and patients with early Parkinson's disease: Evidence for impaired locomotor pattern generation in early Parkinson's disease. Mov Disord 1999:14(4):619625.

16. Morris ME. Movement disorders in people with Parkinson's disease: A model for physical therapy. Phys Ther 2000:80(6): 578-597.

17. Georgiou N, Iansek R, Bradshaw JL, Phillips JG, Mattingley JB, Bradshaw JA. An evaluation of the role of internal cues in the pathogenesis of Parkinsonian hypokinesia. Brain 1993: 116(Pt 6):1175-1187.

18. Hausdorff JM, Schaafsma JD, Balash Y, Bartels AL, Gurevich $\mathrm{T}$, Giladi N. Impaired regulation of stride variability in Parkinson's disease subjects with freezing of gait. Exp Brain Res 2003:149(2):187-194.

19. Nieuwboer A, Dom R, De Weerdt W, Desloovere K, Fieuws $\mathrm{S}$, Broens-Kaucsik E. Abnormalities of the spatiotemporal characteristics of gait at the onset of freezing in Parkinson's disease. Mov Disord 2001:16(6):1066-1075.

20. Hoehn M, Yahr M. Parkinsonism: Onset, progression and mortality. Neurology (Cleveland) 1967:17(5):427-442.

21. Nieuwboer A, De Weerdt W, Dom R, Bogaerts K, Nuyens L. Development of an activity scale for individuals with advanced Parkinson disease: Reliability and "on-off" variability. Phys Ther 2000:80(11):1087-1096.

22. Goetz C, Stebbins GT, Shale HM, Lang AE, Chernik DA, Chmura TA, Ahlskog JE, Dorflinger EE. Utility of an objective Dyskinesia Rating Scale for Parkinson's disease: Inter- and intrarater reliability assessment. Mov Disord 1994:9(4):390-394.

23. Molloy DW, Standish TIM. Mental status and neuropsychological assessment: A guide to the standardized mini-mental state examination. Int Psychogeriatr 1997:9(S1):87-94.

24. Langston JW, Widner H, Goetz C, et al. Core assessment program for intracerebral transplantations (CAPIT). Mov Disord 1992:7(1):2-13.

25. Giladi N, Shabtai H, Simon ES, Biran S, Tal J, Korczyn AD. Construction of freezing of gait questionnaire for patients with Parkinsonism. Parkinsonism Relat Disord 2000:6(3):165170.

26. Sheridan MR, Flowers KA. Movement variability and bradykinesia in Parkinson's disease. Brain 1990:113(Pt 4): $1149-1161$

27. Morris M, Ianesk R, Matyas T, Summers J. Abnormalities in the stride-length-cadence relation in Parkinsonian gait. Mov Disord 1998:13(1):61-69.

28. Schaafsma JD, Balash Y, Gurevich T, Bartels AL, Hausdorff JM, Giladi N. Characterization of freezing of gait subtypes and the response of each to levodopa in Parkinson's disease. Eur J Neurol 2003:10(4):391-398. 